\title{
Particle Production in Two-Photon Collisions at Belle
}

\author{
H. Nakazawa* \\ National Central University (Taiwan) \\ E-mail: nkzwepost.kek. ip
}

\begin{abstract}
We report recent measurements of the $\gamma \gamma \rightarrow \eta \eta$ process in the energy range, $1.096 \mathrm{GeV}<\mathrm{W}<$ $3.8 \mathrm{GeV}$ and in scattering angle, $\left|\cos \theta^{*}\right| \leq 0.9$ or $\leq 1.0$ depending on $W$, where $W$ is the energy of the two-photon center-of-mass system and $\theta^{*}$ is the $\eta$ scattering angle. In the lower energy region, we perform a partial wave analysis to the differential cross section and extract resonance parameters. In the higher energy region, (differential) cross section is compared with QCD predictions. We also present a study of $\eta_{c}(2 S)$ production with 6-prong final states in two-photon collisions.
\end{abstract}

35th International Conference of High Energy Physics - ICHEP2010,

July 22-28, 2010

Paris France

${ }^{*}$ Speaker. 

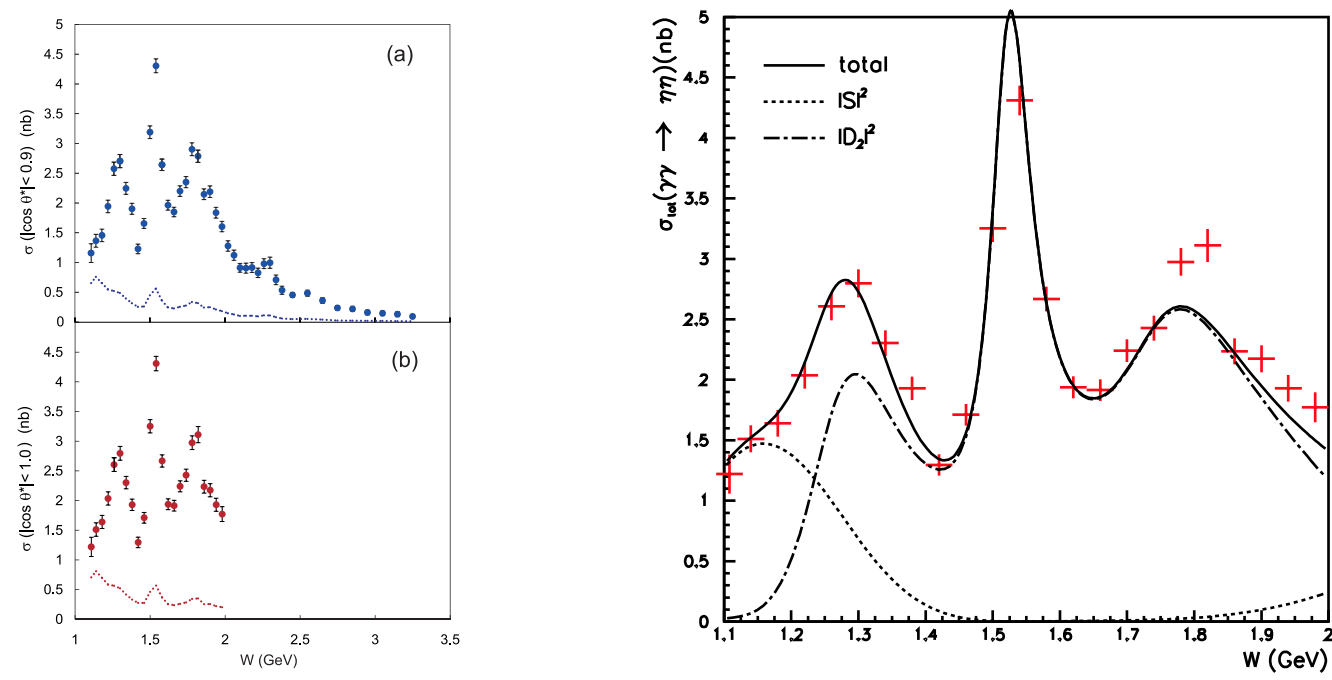

Figure 1: Left: Cross section of the $\gamma \gamma \rightarrow \eta \eta$ process integrated over $\left|\cos \theta^{*}\right|<1(W<2.0 \mathrm{GeV})$ or $\left|\cos \theta^{*}\right|<0.9(W>2.0 \mathrm{GeV})$. Errors are statistical only. The dotted curve shows the size of the systematic uncertainty. Right: The total cross section $\left(\left|\cos \theta^{*}\right|<1.0\right)$ and fitted curves. Dotted (dot-dashed) curves are $|S|^{2}\left(\left|D_{2}\right|^{2}\right)$ from the fit.

\section{Introduction}

Two-photon production of exclusive hadronic final states provides useful information about resonances and pertubative and nonperturbative QCD. From theoretical viewpoint, two-photon process is attractive because of the absence of strong interactions in the initial state and the possibility of calculating $\gamma \gamma \rightarrow q \bar{q}$ amplitudes. In addition, the quantum numbers of the final state are restricted to states of charge conjugation $C=+1$ with $J=1$ forbidden.

We have measured production of charged meson pairs [四], neutral meson pairs [[]], proton antiproton pair [B] and $D$-meson pair [四] in two-photon collisions. This paper reports recent measurements of $\gamma \gamma \rightarrow \eta \eta$ [5] and $\gamma \gamma \rightarrow \eta_{c}(2 S) \rightarrow 6$ prong.

\section{2. $\gamma \gamma \rightarrow \eta \eta$}

The results are based on a $393 \mathrm{fb}^{-1}$ data sample collected with the Belle detector [6] at the KEKB $e^{+} e^{-}$collider [ $[\mathbf{D}] . \eta$ is reconstructed with a photon pair. This pure neutral final states are selected with energy sum and cluster counting triggers, both of which information are provided by a CsI(Tl) electromagnetic calorimeter. We subtract background by studying sideband events in two-dimensional $M_{1}(\gamma \gamma)-M_{2}(\gamma \gamma)$ distributions. Further background effects are studied using $\left|\sum \vec{p}_{t}\right|$ distribution and taken into account as systematic errors. Fig. $($ (Left) shows the total cross sections.

For the lower energy region $1.16 \mathrm{GeV}<\mathrm{W}<2.0 \mathrm{GeV}$, we apply a partial wave analysis to the differential cross section (Fig. $\mathbb{W}$ (Right)). In addition to the known $f_{2}(1270)$ and $f_{2}^{\prime}(1525)$, we introduce a tensor meson $f_{2}(X)$ to describe $D_{2}$ wave, which may correspond to $f_{2}(1810)$ state [ $[8]$, and the mass, width and product of the two-photon decay width and branching fraction $\Gamma_{\gamma \gamma} \mathscr{B}(\eta \eta)$ for $f_{2}(X)$ are obtained to be $1737 \pm 9 \mathrm{MeV} / \mathrm{c}^{2}, 228_{-20}^{+21} \mathrm{MeV}$ and $5.2_{-0.8}^{+0.9} \mathrm{eV}$, respectively. In the 

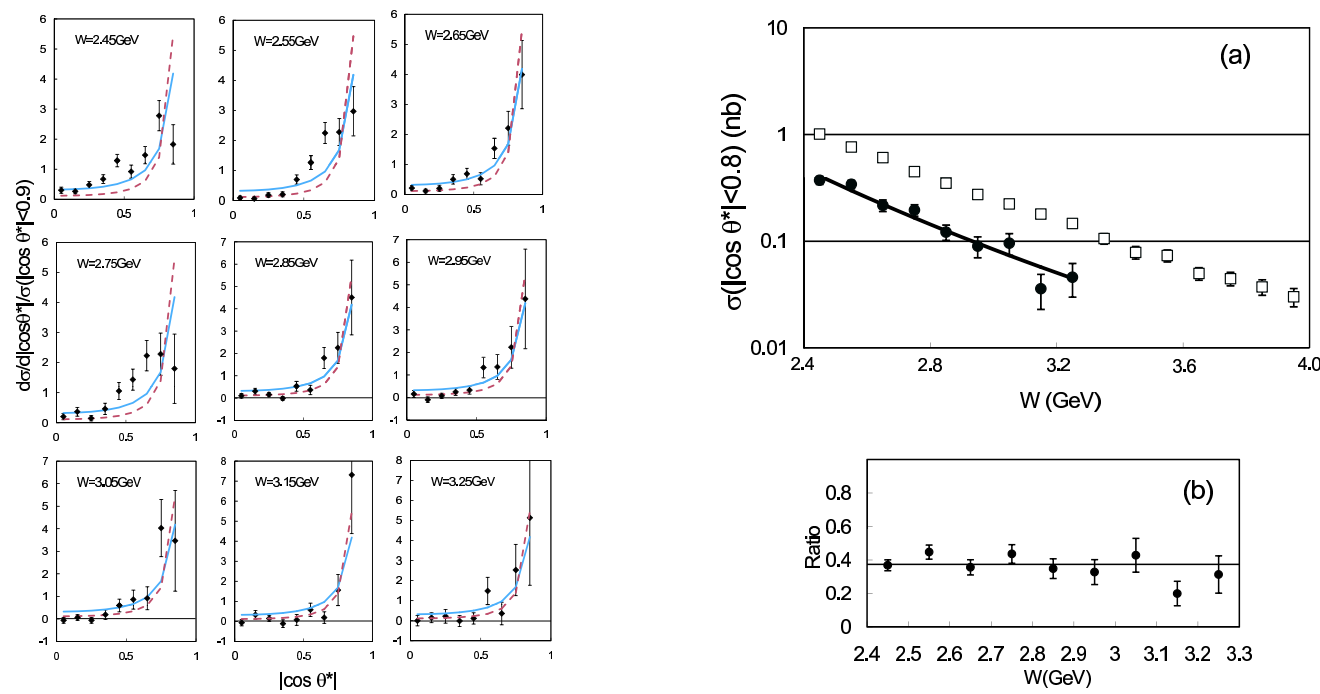

Figure 2: Left: Angular dependence of the differential cross sections in different $W$ regions. The solid and dashed curves are proportional to $1 / \sin ^{4} \theta^{*}$ and $1 / \sin ^{6} \theta^{*}$, respectively. All of them are normalized to have unit area. Right: (a) The $W$ dependence of the cross sections $\left(\left|\cos \theta^{*}\right|<0.8\right)$ for the $\pi^{0} \pi^{0}$ (open squares) and $\eta \eta$ (closed circles) processes. The curve is the power-law fit for $\eta \eta$. (b) The $W$ dependence of the cross section ratio of $\eta \eta$ to $\pi^{0} \pi^{0}\left(\left|\cos \theta^{*}\right|<0.8\right)$. The line is the average in the $2.4-3.3 \mathrm{GeV}$ range.

higher energy region $2.4 \mathrm{GeV}<\mathrm{W}<3.2 \mathrm{GeV}$ where effects from resonances are small, we compare the (differential) cross section with (pertubative) QCD ((p)QCD) predictions. In our previous studies for $\pi^{+} \pi^{-}, K^{+} K^{-}, \pi^{0} \pi^{0}$ and $\eta \pi^{0}$ modes, the angular dependence in $W \gtrsim 3.0 \mathrm{GeV}$ were consistent with $1 / \sin ^{4} \theta^{*}$ while pQCD predicts $1 / \sin ^{4} \theta^{*}$ only for charged meson pair. We find that the angular dependence of $\eta \eta$ is in better agreement with $1 / \sin ^{6} \theta^{*}$ than $1 / \sin ^{4} \theta^{*}$ (Fig. [ (Left)). The total cross section is fitted with a power-low function, $W^{-n}$ and $n=7.8 \pm 0.6 \pm 0.4$ is obtained (Fig. $\mathbb{Z}(\mathrm{a})$ ). Fig. $\mathbb{\nabla}(\mathrm{b})$ shows the $W$ dependence of the ratio between the measured cross section integrated over $\left|\cos \theta^{*}\right|<0.8$ of $\gamma \gamma \rightarrow \eta \eta$ to $\gamma \gamma \rightarrow \pi^{0} \pi^{0}$. The averaged value of $0.37 \pm 0.02 \pm 0.03$ can be compared with the (p)QCD predictions [Q]
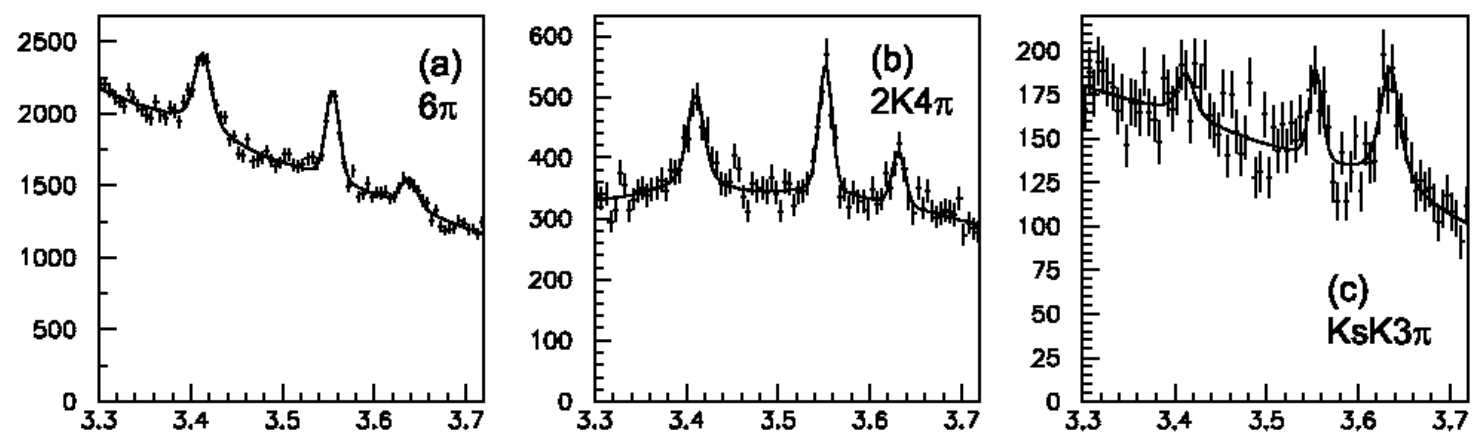

Figure 3: $\chi_{c 0}, \chi_{c 2}$ and $\eta_{c}(2 S)$ peaks in (a) $6 \pi$, (b) $4 K 2 \pi$ and (c) $K_{S} K 3 \pi$ mass distributions. Curves are the best fit results. 
Table 1: Fit results for $\eta_{c}(2 S)$ parameters. Errors are statistical, systematics and effects from possible interference with continuum.

\begin{tabular}{llllll}
\hline \hline Process & $M\left(\mathrm{MeV} / \mathrm{c}^{2}\right)$ & $\Gamma(\mathrm{MeV})$ & evts & signi. & $\Gamma_{\gamma \gamma} \mathscr{B}(\mathrm{eV})$ \\
\hline $6 \pi$ & $3638.9 \pm 1.6 \pm 2.3$ & $10.7 \pm 4.9$ & $1485 \pm 274$ & $8.5 \sigma$ & $20.1 \pm 3.7 \pm 3.2$ \\
\hline $2 K 4 \pi$ & $3634.7 \pm 1.6 \pm 2.8$ & $1.4_{-1.4}^{+6.3}, 13(90 \%$ C.L. $)$ & $407 \pm 91$ & $6.2 \sigma$ & $10.2 \pm 2.3 \pm 3.4$ \\
\hline$K_{S} K 3 \pi$ & $3636.5 \pm 1.8 \pm 2.4$ & $15.9 \pm 5.7$ & $563 \pm 71$ & $8.7 \sigma$ & $30.7 \pm 3.9 \pm 3.7$ \\
\hline Average & $3636.9 \pm 1.1 \pm 2.5 \pm 5.0$ & $9.9 \pm 3.2 \pm 2.6 \pm 2.0$ & & & \\
\hline \hline
\end{tabular}

3. $\gamma \gamma \rightarrow \eta_{c}(2 S)$

Motivated by the fact that $\eta_{c}(2 S)$ was not seen in our result of four-prong final states [ए0], we study six-prong final states with four modes, $\pi^{+} \pi^{-} \pi^{+} \pi^{-} \pi^{+} \pi^{-}(6 \pi), K^{+} K^{-} \pi^{+} \pi^{-} \pi^{+} \pi^{-}(2 K 4 \pi)$, $K^{+} K^{-} K^{+} K^{-} \pi^{+} \pi^{-}(4 K 2 \pi)$ and $K_{S} K^{ \pm} \pi^{\mp} \pi^{+} \pi^{-}\left(K_{S} K 3 \pi\right)$, using a data sample of $923 \mathrm{fb}^{-1} \cdot \chi_{c 0}$, $\chi_{c 2}$ and $\eta_{c}(2 S)$ peaks are clearly seen in $6 \pi, 2 K 4 \pi$ and $K_{S} K 3 \pi$ mass distributions (Fig. (D)). They are the first observations except $\chi_{c 0} \rightarrow 4 K 2 \pi$ mode. We do not take interference effect with continuum into account, which is estimated as systematic error independently. Fit results for the $\eta_{c}(2 S)$ are summarized in Table $\mathbb{\text { m. }}$.

\section{References}

[1] T. Mori, S. Uehara, Y. Watanabe et al. (Belle Collaboration), J. Phys. Soc. Jpn, 76, 074102 (2007). T. Mori et al. (Belle Collaboration), Phys. Rev. D 75, 051101(R) (2007). H. Nakazawa et al. (Belle Collaboration), Phys. Lett. B 615, 39 (2005). K. Abe et al. (Belle Collaboration), Eur. Phys. J. C 32, 323 (2004).

[2] W. T. Chen et al. (Belle Collaboration), Phys. Lett. B 651, 15 (2007). S. Uehara et al. (Belle Collaboration), Phys. Rev. D 78, 052004 (2008). S. Uehara et al. (Belle Collaboration), Phys. Rev. D 79, 052009 (2009). S. Uehara et al. (Belle Collaboration), Phys. Rev. D 80, 032001 (2009).

[3] C. C. Kuo et al. (Belle Collaboration), Phys. Lett. B 621, 41 (2005).

[4] S. Uehara et al. (Belle Collaboration), Phys. Rev. Lett. 96, 082003 (2006).

[5] S. Uehara et al. (Belle Collaboration), hep-ex/1007.3779 (2010), accepted by Phys. Rev. D.

[6] A. Abashian et al. (Belle Collaboration), Nucl. Instr. and Meth. A 479, 117 (2002).

[7] S. Kurokawa and E. Kikutani, Nucl. Instr. and Meth. A 499, 1 (2003).

[8] C. Amsler et al. (Particle Data Group), Phys. Lett. B 667, 1 (2008).

[9] S. J. Brodsky and G. P. Lepage, Phys. Rev. D 24, 1808 (1981). M. Benayoun and V. L. Chernyak, Nucl. Phys. B 329, 285 (1990). M. Diehl, P. Kroll and C. Vogt, Phys. Lett. B 532, 99 (2002). V. L. Chernyak, hep-ph/0912.0623 (2009).

[10] S. Uehara et al. (Belle Collaboration). Eur. Phys. J. C 53, 1 (2008). 